\title{
Treatment Modalities in the Intensive Care Unit for Coronavirus Disease 2019
}

\author{
Anjan Trikha' Akhil Kant Singh ${ }^{1}$ \\ 1Department of Anaesthesiology, Pain Medicine \& Critical Care, \\ All India Institute of Medical Sciences, New Delhi, India
}

Ann Natl Acad Med Sci (India):2020;3:154-160

\begin{abstract}
Address for correspondence Anjan Trikha, MBBS, MD, Department of Anaesthesiology, Pain Medicine \& Critical Care, All India Institute of Medical Sciences, Sri Aurobindo Marg, Ansari Nagar, New Delhi 110029, India (e-mail: anjantrikha@gmail.com).
\end{abstract}

Keywords
- COVID-19
- pneumonia
- viral
- respiratory distress
syndrome
- adult

The last Influenza pandemic of 1918 happened before the advent of modern medicine. We have come a long way since then. But the COVID-19 pandemic has still caught us unprepared on many fronts. The review focuses on the management of critically ill COVID-19 patients and the various treatment modalities being employed to counter this incompletely understood disease.

\section{Introduction}

The world is in the grips of the severe acute respiratory syndrome coronavirus 2 (SARS-CoV2) pandemic. What started as an innocuous viral infection in China in December 2019, has quickly traveled around the world and infected almost all countries on the planet. At the time of writing, there are almost 5 million cases and over 300,000 death attributed to coronavirus disease 2019 (COVID-19). ${ }^{1}$

A large majority of COVID-19 infections are mild and do not require hospitalization. The spectrum of presentations ranges from mild acute respiratory infection to severe acute respiratory tract infections with sepsis and multiple organ system failure. About $5 \%$ of infections require intensive care management. ${ }^{2}$ The case fatality ratio ranges between 4 and $16 \%{ }^{3}$ Most common presenting features are fever, cough, malaise, and breathlessness. The COVID-19 patients may sometimes present with neurological manifestations such as headache, hypogeusia/anosmia, acute cerebrovascular events, seizure, and ataxia. ${ }^{4}$ Older patients have been observed to have hypoxemia without displaying clinical signs of respiratory distress, a condition described as "happy hypoxemia." ${ }^{5}$ Hypertension, diabetes, and preexisting kidney disease are overrepresented in the disease cohort, and obesity has been observed to be an independent risk factor of disease severity ( - Table $\mathbf{1})$ (-9 $^{6-9}$
Patients requiring intensive care management have respiratory failure secondary to COVID-19 viral pneumonia. Viral pneumonia often progresses to acute respiratory distress syndrome, and the disease course may be complicated by myocarditis, acute kidney injury, secondary infections, and sepsis/septic shock. ${ }^{10-13}$

The absence of any proven treatment complicates the issue. The literature abounds with novel experimental therapies and old drugs being repurposed to treat COVID-19. The purpose of this review is to summarize the existent therapies which might be useful in management of COVID-19 patients in the intensive care unit (ICU).

\section{Management of Respiratory Failure}

COVID-19 typically presents with respiratory tract illness of varying degrees which may or may not be associated with fever. The clinical progression is observed to be biphasic. The first phase is characterized by fever, cough, and other constitutional symptoms and is accompanied by radiological worsening during the first week. This is associated with rapid viral replication. By second week, symptoms begin to resolve in most patients. A small subset of patients continues to worsen with radiological and clinical deterioration, with the onset of respiratory failure. 
Table 1 All India Institute Of Medical Sciences New Delhi coronavirus disease data (unpublished)

\begin{tabular}{|l|l|}
\hline Total number of patients & 173 \\
\hline Hypertension & $48(27 \%)$ \\
\hline Chronic Kidney disease & $37(21 \%)$ \\
On maintenance hemodialysis & $12(6.9 \%)$ \\
\hline Diabetes mellitus & $23(13.2 \%)$ \\
\hline
\end{tabular}

The management of respiratory failure in COVID-19 patients are governed by well-established clinical practices with a few important caveats.

\section{Oxygen Therapy and Mechanical Ventilation}

Increasing the oxygen concentration of inhaled air is one of the basic pillars of respiratory failure management. This can be done in several ways. Hypoxic patients are started on oxygen therapy with face masks to target an oxygen saturation $\left(\mathrm{SpO}_{2}\right)$ of greater than $90 \%$. For patients requiring lowflow supplementation, nasal cannula is appropriate. Higher flows may be administered using a simple face mask, venturi device, or nonrebreather mask. One must be vary of the aerosol generating capacity of various therapeutic or diagnostic techniques used commonly in the intensive care unit. Risk of aerosolization increases with the use of higher flows.

\section{Awake Prone Positioning}

When oxygenation does not improve with increasing $\mathrm{FiO}_{2}$, patients are made to lie prone. Proning during invasive mechanical ventilation is advocated for acute respiratory distress syndrome (ARDS) and is almost the standard of care. Prone positioning during severe ARDS improved oxygenation and demonstrated a mortality benefit. ${ }^{14}$ Awake proning works on the same principles and a small, single center study has demonstrated improved oxygenation in COVID-19 patients. ${ }^{15}$ Patient selection is important. Patients-who can communicate and cooperate, change position independently, and have no anticipated difficult airway issues-can be given a trial of awake proning. Patients who require an $\mathrm{FiO}_{2}$ of more than $30 \%$ should be given a trial of this technique. Patients with a P:F ratio of less than 100 do not seem to benefit from awake prone positioning. ${ }^{16}$

Thus, any patient with an $\mathrm{FiO}_{2}$ requirement greater than $30 \%$ and a P:F ratio greater than 100 who can cooperate is a good candidate for awake proning. The procedure should be explained to the patient beforehand. Pillows may be required for comfortable positioning, especially below the chest. Adequate length of circuit tubing should be ensured.

After proning, the patient's saturation should be closely observed to ensure that there is no decrease. If there is no decrease, prone positioning should be maximized as much as possible, preferably for at least 2 hours. If the patient is cooperative, cyclic position change as described in - Fig. 1 can be done. Oxygen saturation should be monitored after every position change and oxygen therapy titrated down as tolerated.

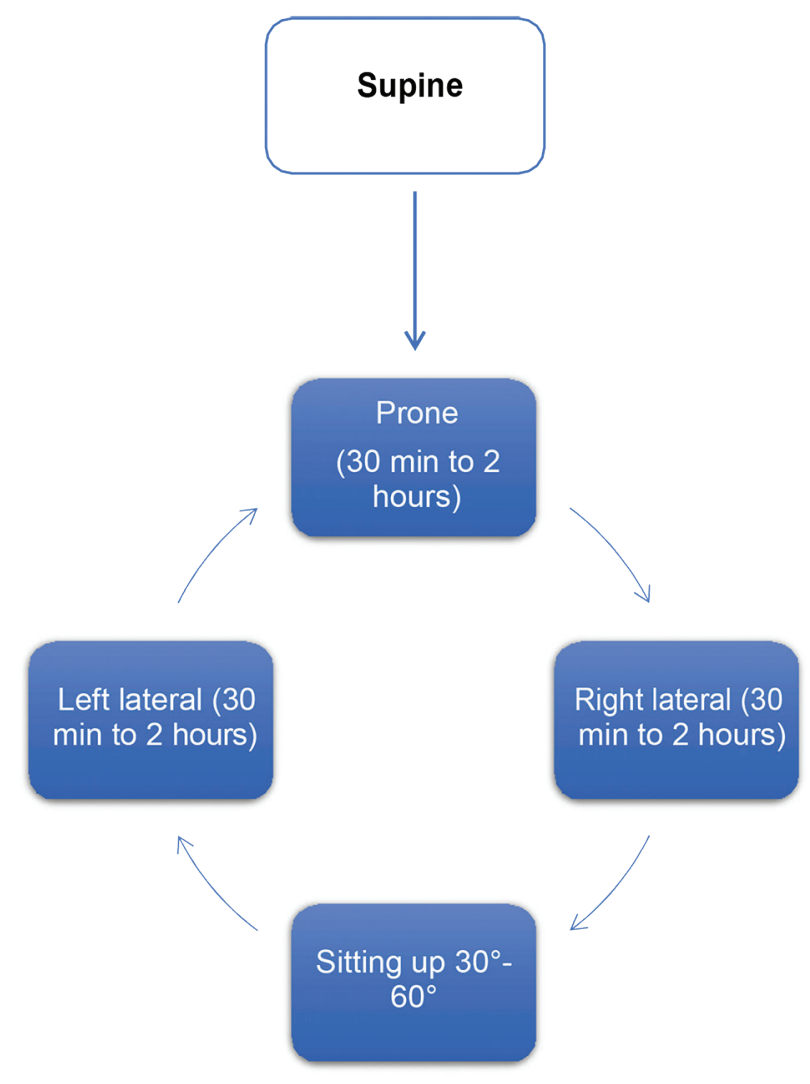

Fig. 1 Awake prone positioning protocol.

If the patient desaturates, looks tired or in distress, or is unable to tolerate position; he or she should be made supine and care escalated as required.

\section{Noninvasive Ventilation and High-Flow Nasal Cannula}

If awake proning does not result in improvement in oxygenation and the patient deteriorates, the patient may be put on either high-flow nasal cannula (HFNC) or noninvasive ventilation (NIV). It is important to understand that both modalities lead to increased aerosolization, consequently increased risk to health care workers, and lead to delay in intubation, which may increase mortality. A school of thought advocates not using these modalities at all in COVID-19 patients. But this viewpoint must be balanced by the availability of resources, both material and human. Intubating all deteriorating patients might be feasible in a setting with adequate resources, but this is not possible in a resource poor scenario. Following, it will result in an undue strain on ventilator demand in case of a surge.

At our institution, HFNC and NIV are important steps on the management ladder, though we prefer NIV over HFNC. The ERS/ATS guidelines recommend the use of NIV as a preventive strategy for avoiding intubation in hypoxemic acute respiratory failure. ${ }^{17,18}$ NIV and HFNC may be an option for mild to moderate ARDS $\left(\mathrm{PaO}_{2} / \mathrm{FiO}_{2}>100\right)$.

Helmet NIV has better acceptance than NIV by facemask. Using a helmet with double limb circuit and a good seal 
at the neck-helmet interface is a safe option in the COVID setting. ${ }^{18,19}$ The use of a "helmet continuous positive airway pressure (CPAP) bundle" has been suggested to improve patient comfort and compliance with the helmet. ${ }^{20}$ We start CPAP with the lowest effective pressures ( 5 to $10 \mathrm{~cm}$ of $\mathrm{H}_{2} \mathrm{O}$ ). For HFNC, we start with low-flow rates $(20 \mathrm{~L} / \mathrm{min})$ and titrate according to the patient's requirement. This strategy allows us to mitigate the risk of aerosolization. HFNC or NIV can also be combined with awake proning resulting in improved oxygenation.

Early application of HFNC or NIV in a patient of moderate ARDS in prone position resulted in avoidance of intubation and improvement in oxygenation. ${ }^{16}$ Presumably, these findings can be extrapolated to COVID-19 respiratory failure as well. One of the drawbacks of using noninvasive modes of ventilation in pursuit of avoidance of intubation is the higher level of vigilance required on part of the health care personnel. Thus, patients on such modalities must be monitored closely with frequent blood gas analysis (every 2-3 hours) to ensure safety. A low threshold for intubation should be maintained.

\section{Invasive Ventilation}

Most COVID-19 patients with severe ARDS will ultimately need invasive mechanical ventilation. Timing of intubation is important. If the patient is on NIV/HFNC, it is imperative that he/she is monitored closely for clinical and/or biochemical deterioration and intubation is not delayed as it is known to increase mortality. Intubation is known to have the highest risk of transmission due to aerosol generation. ${ }^{21}$ It is important that certain pertinent points are kept in mind before performing intubation (- Table 2). ${ }^{22}$

"Aerosol boxes" have been devised to prevent aerosol dissemination during endotracheal intubation. ${ }^{23}$ In our experience, such boxes hinder arm movement and may actually delay intubation. We prefer to use a supranormal dose of succinylcholine or rocuronium, and propofol as part of a Rapid sequence intubation technique and intubate using the precautions mentioned previously.

The primary aim of ventilation strategy in a COVID-19 patient is avoidance of ventilator induced lung injury. That means using a low tidal volume ventilation (LTVV) strategy as described by the ARDS network. ${ }^{24}$ We use tidal volumes $6 \mathrm{~mL} / \mathrm{kg}$ predicted body weight which targets a $\mathrm{P}_{\text {plat }}<30 \mathrm{~cm}$ of $\mathrm{H}_{2} \mathrm{O}$ with the prescribed positive end-expiratory pressure (PEEP). As is the practice in ARDS, ventilation is adjusted to keep driving pressure $\left(\mathrm{DP}=\mathrm{P}_{\text {plat }}-\mathrm{PEEP}\right)$ less than $15 \mathrm{~cm}$ of $\mathrm{H}_{2} \mathrm{O}$.

The mode of ventilation used varies, but a volume limited assist control mode is the most used mode. This mode provides a consistent tidal volume and is less prone to breath-to-breath changes in the respiratory system compliance, which might lead to unstable volumes being delivered. In the case of pressure limited modes, changes in compliance can lead to breath to breath variability in delivered tidal volume. Delivery of LTVV requires deep sedation, and the higher than normal rates can lead to generation of auto-PEEP. Ventilator dyssynchrony is common and may occur in up to $25 \%$ of ventilated patients ${ }^{25,26}$ and includes
Table 2 Important issues to remember during intubation

Things to keep in mind

- An intubation checklist should be followed to avoid confusion

- The most qualified individual (e.g., anesthesiologist) should perform the intubation to decrease the number of attempts

- If possible, the intubation should be performed in an airborne infection isolation room

- Minimum number of personnel should be in the room during the procedure

- Personnel involved in intubation should be properly donned with personnel protective equipment which includes a fit tested N-95 mask, eye protection, cap, gown shoe covers, and gloves

- Preoxygenation should be performed using a $100 \%$ nonrebreather masks. Bag mask ventilation should be avoided to prevent aerosol generation. If the need for assisting ventilation is unavoidable, the mask should be replaced with a supraglottic device

- An heat and moisture exchangers filter should be placed between the mask/supraglottic device and circuit

- Rapid sequence induction should be planned to decrease the apneic duration and to ensure complete paralysis at the time of intubation

- Video laryngoscope should be used to perform the intubation. This improves first pass success and allows distancing between the patient and the physician

double triggering, ineffective triggering and flow dyssynchrony. Various maneuvers and changes to the ventilatory settings can be performed to address these problems.

If these maneuvers fail to rectify dyssynchrony, the ventilatory modes can be changed. There are various options such as pressure-regulated volume control (PRVC), pressure support, airway pressure release ventilation (APRV), volume-targeted pressure-controlled ventilation (e.g., VC plus), and neurally adjusted ventilatory assist (NAVA) mode. We will briefly describe PRVC and APRV modes.

\section{Pressure-Regulated Volume Control}

In PRVC, the tidal volume is set and the inspiratory pressure changes to attain the target tidal volume. The inspiratory pressure supplied depends on the pressure required to attain the tidal volume during the previous breath. This is a time cycled mode of ventilation. Thus, the duration of inspiration depends on the respiratory rate and the I:E ratio.

\section{Airway Pressure Release Ventilation}

During APRV, a high continuous positive airway pressure ( $\mathrm{P}$ high) is delivered for a long duration (T high) and then falls to a lower pressure (P low) for a shorter duration (T low). The 
transition from $\mathrm{P}$ high to $\mathrm{P}$ low deflates the lung and inflates the lung when $\mathrm{P}$ low transitions to $\mathrm{P}$ high. The long duration at $\mathrm{P}$ high increases alveolar recruitment. ${ }^{27,28}$ Driving pressure is the difference between P high and P low, and tidal volume is related to the driving pressure and the compliance. APRV has been shown to decrease days on ventilator, days in the ICU, sedation, and muscle relaxant requirement, but it had no effect on the mortality. ${ }^{29}$

Prone positioning has a proven benefit in ARDS and maybe especially beneficial in COVID-19 ARDS given the greater propensity of peripheral and dorsal areas of the lungs to be affected. ${ }^{30}$ It is the logical next step when LTVV fails to improve the oxygenation. In our practice, we prone patients for a minimum of 14 hours a day. Contraindications and complications remain the same as for any other ARDS case. Care should be taken to avoid venting of circuit to air which can be prevented by clamping it during disconnections. The PROSEVA trial demonstrated that the benefit from proning was accrued when it was done early rather than late. ${ }^{14}$

The decision to stop proning a patient must be individualized. $\mathrm{PaO}_{2} / \mathrm{FiO}_{2}>150$ on a $\mathrm{FiO}_{2}<0.6$ and PEEP $<10 \mathrm{~cm}$ of $\mathrm{H}_{2} \mathrm{O}$ for at least 4 hours after the last prone session makes for a good candidate for ceasing prone positioning.

Neuromuscular blockade is not used routinely because of concerns for critical illness polyneuromyopathy. It should be used when there is refractory hypoxemia or patient ventilator dyssynchrony.

\section{Extracorporeal Membrane Oxygenation}

Veno-venous-ECMO (VV-ECMO) is reserved for the most severe form of ARDS, and maybe suitable for patients who have failed standard low-volume tidal ventilation strategies and/or who have failed or cannot undergo prone ventilation and high PEEP strategies. Few centers perform ECMO on a regular basis, especially so in medium- to low-income countries and it is a resource intensive procedure. A cohort study of ECMO database of patients with H1N1-related ARDS showed that hospital mortality rate was $23.7 \%$ for ECMO-referred patients versus $52.5 \%$ for non-ECMO referred patients. ${ }^{31}$ Retrospective data from ECMO usage in patients with middle eastern respiratory syndrome (MERS) also demonstrated benefit. $^{32}$ On the flipside, experience from China has so far not demonstrated conclusive benefit from the use of ECMO in COVID-19 patients. $^{33}$ In low-resource settings, thought should be given to the dilemma of providing an advanced and expensive, yet unproven therapy to a few patients versus routine care to many patients. We do not provide ECMO as a routine therapy for COVID-19 ARDS patients at our institution.

\section{Fluid Management}

We prefer to use a conservative fluid management strategy as is advised for any ARDS patient unless the patient has sepsis or volume depletion secondary to gastrointestinal losses, fever, etc. ${ }^{34}$ Management of COVID positive patients who have septic shock is similar to patients having septic shock due to other causes. In the recovering COVID-19 patient on the ventilator, we prefer to keep them "dry" on the day before planned extubation. In our experience, this facilitates the weaning process. Colloids like starches and gelatin or hypotonic crystalloids are no longer recommended for use in the intensive care unit. If a colloid has to be used when patients require substantial amounts of crystalloid, albumin can be used.

\section{Antibiotics}

All our patients receive empirical broad-spectrum antibiotic coverage as it is common to have superimposed bacterial infection, especially in the presence of comorbidities. The specific antibiotic coverage can be tailored according to the local infectious disease epidemiology. ${ }^{35}$ In the presence of local seasonal influenza, a neuraminidase inhibitor (e.g., Oseltamivir) may be added.

\section{Thromboprophylaxis}

Routine thromboprophylaxis is warranted in all patients receiving mechanical ventilation in the absence of any contraindications. ${ }^{36}$ This recommendation is valid for COVID-19 patients as well. Anecdotal evidence and local guidelines at various hospitals across the world suggests that physicians consider COVID-19 patients to be at a higher risk of venous thromboembolism..$^{37}$ This is reflected in the adoption of an intermediate intensity (i.e., administering the usual daily low molecular weight heparin (LMWH) dose twice daily) or even a therapeutic intensity dosing strategy.

We prefer to administer the standard prophylactic once daily dosing of LMWH and instituting therapeutic LMWH dosing if there is evidence of any venous thrombosis or signs of cytokine storm syndrome. Care should be taken to consider the patient's renal function while selecting the agent and the dose, and to individualize anticoagulation.

If the patient is on warfarin at admission (mechanical heart valves, atrial fibrillation, etc.), it should be continued.

In case of any contraindications to pharmacological prophylaxis, mechanical thromboprophylaxis should be used.

\section{Supportive Therapies}

At the time of writing this review, there has been no therapeutic agent approved for use in COVID-19 patients. There has been interest in several potential agents and trials are ongoing.

\section{Corticosteroids}

Usage of systemic corticosteroids in MERS resulted in increased viral shedding, delayed viral clearance, and increased days on ventilator and mortality. ${ }^{38-41}$ The World Health Organization, the Society for Critical Care Medicine, and the Infectious Disease Society of America recommend against the routine use of systemic corticosteroids in all COVID-19 positive patients. ${ }^{34,35,42}$ If the patient has underlying chronic obstructive pulmonary disease or asthma, in septic shock, or has severe ARDS; corticosteroids should be used. ${ }^{34}$ Corticosteroids may also be used in severe COVID-19 with cytokine release syndrome (CRS). We administer methylprednisolone $2 \mathrm{mg} / \mathrm{kg} /$ day for 5 days as mandated by government guidelines. ${ }^{43}$ 


\section{Hydroxychloroquine/Chloroquine}

Hydroxychloroquine was developed and subsequently approved for the treatment of malaria in 1955 while chloroquine was developed in the 1930s. The mechanism of action is believed to be accumulation within lysosomes and alteration of the internal $\mathrm{pH}$. Both hydroxychloroquine (HCQS) and chloroquine inhibit SARS-CoV2 in vitro, but there is limited and good quality clinical data which show a clear benefit. The U.S. Food and Drug Administration (FDA) has issued an emergency use authorization while most clinical societies discourage use outside of a clinical trial.

The most concerning side effect of HCQS is QT prolongation and should be avoided in patients having QTC prolongation at baseline, or on other drugs causing conduction abnormalities.

We administer hydroxychloroquine $400 \mathrm{mg}$ every 12 hours on the first day, followed by 400 mg daily for 5 days.

A recent observational study has allayed fears to some extent about the side effects of hydroxychloroquine usage in COVID-19 patients. ${ }^{44}$ But in the absence of robust data, it is not recommended to use HCQS in all COVID-19 patients.

Azithromycin, a macrolide antibiotic has known immunomodulatory properties. ${ }^{45}$ When combined with HCQS, it is thought to have a synergistic action on viral activity. Caution should be exercised when combining both drugs as azithromycin also causes QT prolongation.

\section{Interleukin-6 Antagonists}

Drugs such as tocilizumab, sarilumab, and siltuximab are interleukin (IL)-6 antagonists. Tocilizumab has been approved as a therapy for CRS related to CAR-T cell therapy. Since CRS is a common feature of severe COVID-19 infections (presence of persistent fevers, elevated IL-6 and other cytokines, and elevated ferritin, D-dimer, and other inflammatory markers), it follows that tocilizumab and other IL- 6 antagonists have a role to play. Indeed, case reports and observational studies have described the use of tocilizumab in severe COVID19 patients. The U.S. FDA has recently approved a phase III trial for tocilizumab usage in COVID-19 and multiple RCTs are ongoing to answer questions about its efficacy.

\section{Ivermectin}

Ivermectin is an FDA-approved and broad spectrum antiparasitic agent that has been shown to have antiviral activity against a broad range of viruses, including SARS-CoV2 in vitro. ${ }^{46}$ Indeed, a case has been made for the synergistic action of hydroxychloroquine and ivermectin in COVID-19 infections ${ }^{47}$ More robust clinical data are still awaited before use in COVID-19 can be recommended.

\section{Remdesivir}

Remdesivir is a nucleotide analog that has in vitro activity against SARS-CoV2. ${ }^{48}$ The U.S. FDA granted emergency use authorization for remdesivir for children and adults with severe COVID-19 but is not available in India yet. There are ongoing trials to ascertain its efficacy in treating COVID-19 with the current evidence inconclusive. Preliminary results demonstrate a probable efficacy in treating COVID-19 infections, but the target patient subset is unclear.

Remdesivir is not recommended in patients with alanine aminotransferase (ALT) levels more than five times the upper limit of normal. The drug should be discontinued if these ALT levels are breached. The drug should not be given in patients with an estimated glomerular filtration rate $<30 \mathrm{~mL} / \mathrm{min}$ per $1.73 \mathrm{~m}^{2}$.

\section{Lopinavir-Ritonavir}

It is a combined protease inhibitor, primarily used for HIV, which has in vitro activity against SARS-CoV. ${ }^{49}$ It appears to have minimal activity against SARS-CoV2.

Cao et al reported no significant difference in time to clinical improvement, reduction in viral load, or 28-day mortality with lopinavir-ritonavir compared with standard care in patients with severe COVID-19.

The use of lopinavir-ritonavir outside the context of a clinical trial is not recommended.

\section{Convalescent Plasma}

Convalescent plasma is plasma prepared from a patient who has recovered from an illness. It is essentially a way to transfer passive immunity to a sick patient. A systematic review to assess the effectiveness of this therapy in severe acute respiratory illness of viral etiology concluded that convalescent plasma was effective in reducing mortality. ${ }^{50}$

Whether a recovered patient can donate plasma is dependent on several factors such as consent for the procedure, blood type matching, antibody titers, and absence of transmissible infections.

Ideally, convalescent plasma should be administered in the early stages of the disease when the viral inoculum is low. Possibility of adverse effects such as volume overload, transfusion reactions, antibody dependent enhancement of infections, etc. should also be considered.

\section{Plasmapheresis}

While targeting the infectious agent, that is, the virus is inarguably important, mitigation of the excessive host response is also a therapeutic target. The host response is made up of a complex interplay between excessive cytokine release, endothelial dysfunction, and hypercoagulability. ${ }^{51,52}$ Plasmapheresis or therapeutic plasma exchange (TPE) acts on multiple levels of the inflammatory cascade to mitigate the exaggerated immune response..$^{53}$

Although just a single center and retrospective study, this study showed a mortality benefit when TPE was used versus standard care in the subset where pneumonia was the source of sepsis. ${ }^{54}$ Similarly, Ma et al reported their encouraging experience of using TPE in patients with severe COVID-19 infections with cytokine storm. ${ }^{55}$

Plasmapheresis seems to be a promising option in COVID-19 and awaits more robust data.

\section{Blood Purification Devices}

Several blood purification devices are available on the market that can remove both endogenous and exogenous 
inflammatory mediators. The three most well known of these devices are Oxiris, Toraymyxin, and Cytosorb. All three have varying efficacy and adsorption capacity for the removal of cytokines and inflammatory mediators. ${ }^{56}$

The usage of such devices requires specialized equipment and expensive consumables and have unproven efficacy in the management of COVID-19. Their routine usage cannot be recommended at this time.

\section{Conclusion}

The present review describes in brief the current therapeutic landscape for COVID-19.

Unfortunately, there is no one agent which can be said to be a "silver bullet" against COVID-19. While the basics of management of respiratory failure remain the same, we are still lacking any specific therapies. Ongoing research on various agents and modalities will shed more light on the treatment of this pandemic.

\section{Conflict of Interest}

None declared.

\section{References}

1 Coronavirus Update (Live). 4,924,208 cases and 320,807 deaths from COVID-19 virus pandemic - Worldometer. Available at: https://www.worldometers.info/coronavirus/. Accessed May 19,2020

2 Wu Z, McGoogan JM. Characteristics of and important lessons from the coronavirus disease 2019 (COVID-19) outbreak in China: summary of a report of 72314 cases from the chinese center for disease control and prevention. JAMA 2020;323(13):1239-1242

3 Mortality Analyses. Johns Hopkins Coronavirus Resource Center. Available at: https://coronavirus.jhu.edu/data/mortality. Accessed May 7, 2020

4 Wilson MP, Jack AS. Coronavirus disease 2019 (COVID-19) in neurology and neurosurgery: a scoping review of the early literature. Clin Neurol Neurosurg 2020;193:105866

5 Couzin-Frankel J. The mystery of the pandemic's 'happy hypoxia' Science 2020;368(6490):455-456

6 Simonnet A, Chetboun M, Poissy J, et al; LICORN and the Lille COVID-19 and Obesity study group. High prevalence of obesity in severe acute respiratory syndrome coronavirus-2 (SARS-CoV-2) requiring invasive mechanical ventilation. Obesity (Silver Spring) 2020;28(7)1195-1199

7 Richardson S, Hirsch JS, Narasimhan M, et al; and the Northwell COVID-19 Research Consortium. Presenting characteristics, comorbidities, and outcomes among 5700 patients hospitalized with COVID-19 in the New York City area. JAMA 2020;323(20):2052-2059

8 Grasselli G, Zangrillo A, Zanella A, et al; COVID-19 Lombardy ICU Network. Baseline characteristics and outcomes of 1591 patients infected with SARS-CoV-2 admitted to ICUs of the Lombardy region, Italy. JAMA 2020;323(16):1574-1581

9 Xie J, Tong Z, Guan X, Du B, Qiu H, Slutsky AS. Critical care crisis and some recommendations during the COVID-19 epidemic in China. Intensive Care Med 2020;46(5):837-840

10 Chen N, Zhou M, Dong X, et al. Epidemiological and clinical characteristics of 99 cases of 2019 novel coronavirus pneumonia in Wuhan, China: a descriptive study. Lancet 2020;395(10223):507-513
11 Huang C, Wang Y, Li X, et al. Clinical features of patients infected with 2019 novel coronavirus in Wuhan, China. Lancet 2020;395(10223):497-506

12 Guan WJ, Ni ZY, Hu Y, et al; China Medical Treatment Expert Group for Covid-19. Clinical characteristics of coronavirus disease 2019 in China. N Engl J Med 2020;382(18):1708-1720

13 Wang L-Z, Hu X-X, Liu X, Qian P, Ge J-M, Tang B-L. Influence of epidural dexamethasone on maternal temperature and serum cytokine concentration after labor epidural analgesia. Int J Gynaecol Obstet 2011;113(1):40-43

14 Guérin C, Reignier J, Richard J-C, et al; PROSEVA Study Group. Prone positioning in severe acute respiratory distress syndrome. N Engl J Med 2013;368(23):2159-2168

15 Caputo ND, Strayer RJ, Levitan R. Early self-proning in awake, non-intubated patients in the emergency department: a single ED's experience during the COVID-19 pandemic. Acad Emerg Med 2020;27(5):375-378

16 Ding L, Wang L, Ma W, He H. Efficacy and safety of early prone positioning combined with HFNC or NIV in moderate to severe ARDS: a multi-center prospective cohort study. Crit Care 2020;24(1):28

17 Rochwerg B, Brochard L, Elliott MW, et al. Official ERS/ATS clinical practice guidelines: noninvasive ventilation for acute respiratory failure. Eur Respir J 2017;50(2):1602426

18 Cabrini L, Landoni G, Zangrillo A. Minimise nosocomial spread of $2019-\mathrm{nCoV}$ when treating acute respiratory failure. Lancet 2020;395(10225):685

19 Winck JC, Ambrosino N. COVID-19 pandemic and non invasive respiratory management: every goliath needs a David. An evidence based evaluation of problems. Pulmonology 2020;S2531-0437(20):30093-3

20 Lucchini A, Giani M, Isgrò S, Rona R, Foti G. The "helmet bundle" in COVID-19 patients undergoing non invasive ventilation. Intensive Crit Care Nurs 2020;58:102859

21 Tran K, Cimon K, Severn M, Pessoa-Silva CL, Conly J. Aerosol generating procedures and risk of transmission of acute respiratory infections to healthcare workers: a systematic review. PLoS One 2012;7(4):e35797

22 Orser BA. Recommendations for endotracheal intubation of COVID-19 patients. Anesth Analg 2020;130(5):1109-1110

23 Canelli R, Connor CW, Gonzalez M, Nozari A, Ortega R. Barrier enclosure during endotracheal intubation. $\mathrm{N}$ Engl J Med 2020;382(20):1957-1958

24 Brower RG, Matthay MA, Morris A, Schoenfeld D, Thompson BT, Wheeler A; Acute Respiratory Distress Syndrome Network Ventilation with lower tidal volumes as compared with traditional tidal volumes for acute lung injury and the acute respiratory distress syndrome. N Engl J Med 2000;342(18):1301-1308

25 de Wit M, Pedram S, Best AM, Epstein SK. Observational study of patient-ventilator asynchrony and relationship to sedation level. J Crit Care 2009;24(1):74-80

26 Thille AW, Rodriguez P, Cabello B, Lellouche F, Brochard L. Patient-ventilator asynchrony during assisted mechanical ventilation. Intensive Care Med 2006;32(10):1515-1522

27 Stock MC, Downs JB, Frolicher DA. Airway pressure release ventilation. Crit Care Med 1987;15(5):462-466

28 Downs JB, Stock MC. Airway pressure release ventilation: a new concept in ventilatory support. Crit Care Med 1987;15(5):459-461

29 Putensen C, Zech S, Wrigge H, et al. Long-term effects of spontaneous breathing during ventilatory support in patients with acute lung injury. Am J Respir Crit Care Med 2001;164(1):43-49

30 Phua J, Weng L, Ling L, et al; Asian Critical Care Clinical Trials Group. Intensive care management of coronavirus disease 2019 (COVID-19): challenges and recommendations. Lancet Respir Med 2020;8(5):506-517 
31 Noah MA, Peek GJ, Finney SJ, et al. Referral to an extracorporeal membrane oxygenation center and mortality among patients with severe 2009 influenza A(H1N1). JAMA 2011;306(15):1659-1668

32 Alshahrani MS, Sindi A, Alshamsi F, et al. Extracorporeal membrane oxygenation for severe Middle East respiratory syndrome coronavirus. Ann Intensive Care 2018;8(1):3

33 Hong X, Xiong J, Feng Z, Shi Y. Extracorporeal membrane oxygenation (ECMO): does it have a role in the treatment of severe COVID-19? Int J Infect Dis 2020;94:78-80

34 Alhazzani W, Møller MH, Arabi YM, et al. Surviving sepsis campaign: guidelines on the management of critically ill adults with Coronavirus Disease 2019 (COVID-19). Intens Care Med 2020;46(5):854-887

35 Clinical management of severe acute respiratory infection when COVID-19 is suspected. Available at: https:// www.who.int/publications-detail/clinical-management-ofsevere-acute-respiratory-infection-when-novel-coronavirus-(ncov)-infection-is-suspected. Accessed May 8, 2020

36 Rhodes A, Evans LE, Alhazzani W, et al. Surviving sepsis campaign: international guidelines for management of sepsis and septic shock: 2016. Crit Care Med 2017;45(3):486-552

37 COVID-19 and VTE-Anticoagulation - Hematology.org. Available at: https://www.hematology.org:443/covid-19/ covid-19-and-vte- anticoagulation. Accessed May 8, 2020

38 Rodrigo C, Leonardi-Bee J, Nguyen-Van-Tam JS, Lim WS. Effect of corticosteroid therapy on influenza-related mortality: a systematic review and meta-analysis. J Infect Dis 2015;212(2):183-194

39 Corman VM, Albarrak AM, Omrani AS, et al. Viral shedding and antibody response in 37 patients with middle east respiratory syndrome coronavirus infection. Clin Infect Dis 2016;62(4):477-483

40 Arabi YM, Mandourah Y, Al-Hameed F, et al; Saudi Critical Care Trial Group. Corticosteroid therapy for critically ill patients with middle east respiratory syndrome. Am J Respir Crit Care Med 2018;197(6):757-767

41 Zumla A, Hui DS, Perlman S. Middle East respiratory syndrome. Lancet 2015;386(9997):995-1007

42 Infectious Diseases Society of America Guidelines on the Treatment and Management of Patients with COVID-19. Available at: https://www.idsociety.org/practice-guideline/ covid-19-guideline-treatment-and- management/. Accessed May 8, 2020

43 Revised National Clinical Management Guideline for COVID 1931032020.pdf. Available at: https://www.mohfw.gov.in/ pdf/RevisedNationalClinicalManagementGuidelineforCOVI D1931032020.pdf. Accessed May 10, 2020
44 Geleris J, Sun Y, Platt J, et al. Observational study of hydroxychloroquine in hospitalized patients with COVID-19. N Engl J Med 2020;382(25):2411-2418

45 Amsden GW. Anti-inflammatory effects of macrolides-an underappreciated benefit in the treatment of community-acquired respiratory tract infections and chronic inflammatory pulmonary conditions? J Antimicrob Chemother 2005;55(1):10-21

46 Caly L, Druce JD, Catton MG, Jans DA, Wagstaff KM. The FDAapproved drug ivermectin inhibits the replication of SARSCoV-2 in vitro. Antiviral Res 2020;178:104787

47 Patrì A, Fabbrocini G. Hydroxychloroquine and ivermectin: a synergistic combination for COVID-19 chemoprophylaxis and treatment? J Am Acad Dermatol 2020;82(6):e221

48 Sheahan TP, Sims AC, Graham RL, et al. Broad-spectrum antiviral GS-5734 inhibits both epidemic and zoonotic coronaviruses. Sci Transl Med 2017;9(396):eaal3653

49 Cao B, Wang Y, Wen D, et al. A trial of lopinavir-ritonavir in adults hospitalized with severe COVID-19. N Engl J Med 2020;382(19):1787-1799

50 Mair-Jenkins J, Saavedra-Campos M, Baillie JK, et al; Convalescent Plasma Study Group. The effectiveness of convalescent plasma and hyperimmune immunoglobulin for the treatment of severe acute respiratory infections of viral etiology: a systematic review and exploratory meta-analysis. J Infect Dis 2015;211(1):80-90

51 Johansson PI, Stensballe J, Ostrowski SR. Shock induced endotheliopathy (SHINE) in acute critical illness - a unifying pathophysiologic mechanism. Crit Care 2017;21(1):25

52 Nguyen TC, Carcillo JA. Bench-to-bedside review: thrombocytopenia-associated multiple organ failure-a newly appreciated syndrome in the critically ill. Crit Care 2006;10(6):235

53 Knaup H, Stahl K, Schmidt BMW, et al. Early therapeutic plasma exchange in septic shock: a prospective open-label nonrandomized pilot study focusing on safety, hemodynamics, vascular barrier function, and biologic markers. Crit Care 2018;22(1):285

54 Keith P, Day M, Perkins L, Moyer L, Hewitt K, Wells A. A novel treatment approach to the novel coronavirus: an argument for the use of therapeutic plasma exchange for fulminant COVID-19. Crit Care 2020;24(1):128

55 Ma J, Xia P, Zhou Y, et al. Potential effect of blood purification therapy in reducing cytokine storm as a late complication of critically ill COVID-19. Clin Immunol 2020;214:108408

56 Malard B, Lambert C, Kellum JA. In vitro comparison of the adsorption of inflammatory mediators by blood purification devices. Intensive Care Med Exp 2018;6(1):12 